\title{
Effect of Horticultural Occupation Therapy Program on the Emotional Rehabilitation of Acute and Chronic Mental Disorder Patients
}

\author{
Mi Young Kim ${ }^{1}$ and Woo Kwon Park ${ }^{2}$ * \\ 'Department of Occupational Therapy, Seoul Metropolitan Eunpyeong Hospital, Seoul 03476, South Korea \\ ${ }^{2}$ Department of Occupational Therapy, Kyungbok University, Gyeonggido 11138, South Korea
}

\begin{abstract}
This study aimed to analyze the effects of a horticultural occupation therapy program using a healing garden on the emotional rehabilitation of patients with acute and chronic mental disorders, and to identify the possibility of using horticultural occupational therapy as a mental rehabilitation service to promote the social rehabilitation of patients with mental disorders. In this study, 20 patients diagnosed as chronic mental disorder at Seoul Metropolitan E hospital and 22 as acute mental disorder at Incheon $\mathrm{H}$ hospital were divided into 2 groups for Horticultural occupation therapy (HOT) and Group occupational therapy (GOT). General occupational therapy was provided 4 times a week for the experimental groups of patients with chronic and acute mental disorders, and a horticultural occupational therapy program using a healing garden was provided only once a week, a total of 12 sessions, for the experimental groups. For the control groups of patients with acute and chronic mental disorders, occupational therapy was provided 5 times a week only. Among the acute mental disorder patients, the treatment group showed a significant decrease in Beck Depression Inventory (BDI), Beck Anxiety Inventory (BAI) and emotional stress scale (ES), whereas the control group didn't $(p<.001)$. On the other hand, such significant changes were not seen among the chronic GOT. Futhermore, the chronic HOT showed the significant effect in physical stress scale (PSE).
\end{abstract}

Keywords: anxiety, depression, stress

\section{Introduction}

Patients with acute mental disorders are those who have mental disorders for more than 6 months but less than 2 years, and patients with chronic mental disorders are those who have severe symptoms that can deteriorate their social skills and develop adjustment disorders for at least more than 2 years. Patients with mental disorders show impairments in maintaining personal hygiene and cleanliness, and interpersonal relationships in their workplace, school and social life, and need to be hospitalized for a long time and to receive psychiatric treatment (Goldman et al., 1981). Most mentally ill patients have a low confidence and are vulnerable to stress. They have a lack of skills required for daily life, and difficulties in building interpersonal relationships, and experience loneliness, frustration and depression, which, in turn, reduces the quality of life (Liberman, 1987). Drug treatment is an important means for mentally ill patients, but taking

Received: March 28, 2017, Revised: December 30, 2017, Accepted: February 4, 2018

First author: Mi Young Kim, E-mail: shilo@seoul.go.kr, ORCID: 0000-0003-3622-8528

*Corresponding author: Woo Kwon Park, E-mail: wkpark1002@hanmail.net, ORCID: 0000-0002-3438-7843 
antipsychotic drugs is not enough to reduce a sense of boredom, demotivation, anxiety, depression and physical symptoms (Park, 1995). Efforts have been expanded to rehabilitate patients who have chronic mental disorders for more than 2 years. The number of local mental health centers and social adaptation programs for patients with chronic mental disorders has been also increasing. However, it is also necessary to make efforts to deinstitutionalize those with chronic mental disorders. Patients with acute mental disorders have many problems associated with stress, and emergency aid programs have been provided for those who show temporary disorders after experiencing social disasters or accidents. Meyer (1922) found that mentally ill patents often face difficulties in adapting to social environments due to stress, and highlighted the importance of work through which patients can do meaningful activities, spend time properly and have a sense of satisfaction in order for them to overcome the difficulties. Chu (2007) pointed out that social rehabilitation programs for mentally ill patients need to focus on improving psychological and mental elements, such as anxiety, depression, self-esteem and the restoration of personal relationships, as well as building vocational skills simultaneously. Relf(1990) explained that horticultural therapy increases the will to live and helps rehabilitate and restore both physical and mental health through emotional experiences and activities of growing and taking care of plants. Since there are limitations in treating patients with mental disorders only with drug treatment, research has been actively conducted on horticultural activities that are known to supplement drug treatment and to be effective in improving emotional stability. Horticultural activities have been found to reduce stress that patients with mental disorders experience (Doxon et al., 1987), to be effective in improving self-esteem and interpersonal relationships and reducing depression, and to reduce negativity toward others (Lee et al., 2006). They are also effective in improving the self-esteem and sociality of patients (Um, 2000).

Recently, horticultural activities have been performed for various target groups, and healing gardens have emerged as a therapeutic space focusing on dynamic activities and accessibility. A healing garden is a space that increases participants's involvement in social activities and rehabilitation and reduces their stress through contact with nature (Marcus and Barnes, 1999). In this regard, this study aimed to analyze the effects of a horticultural occupation therapy program using a healing garden on the emotional rehabilitation of patients with acute and chronic mental disorders, and to identify the possibility of using horticultural occupational therapy as a mental rehabilitation service to promote the social rehabilitation of patients with mental disorders.

\section{Research Methods}

\section{Research subjects}

This study was conducted on a total of 42 patients: 20 patients who were hospitalized for chronic mental disorders in $\mathrm{E}$ municipal hospital in Seoul, and 22 patients who were hospitalized for acute mental disorders in $\mathrm{H}$ hospital in Incheon from March to August, 2010. Research subjects were selected based on the following criteria:

1. Patients who were diagnosed with a mental disorder by a psychiatrist according to the guidelines of the International Classification of Diseases (ICD-10)

2. Patients with acute mental disorders who were diagnosed with the illness less than one year ago

3. Patients with chronic mental disorders who were diagnosed with the illness earlier than 2 years ago

4. Patients who were informed of the purpose of this study and consented to participate in this study 


\section{Research methods}

In this study, 20 patients with chronic mental disorders were divided into the experimental group (10 patients) and the control group (10 patients) as they preferred regardless of their gender and age, and 22 patients with acute mental disorders were also divided into the experimental group (11 patients) and the control group (11 patients) as they preferred regardless of their gender and age. General occupational therapy was provided 4 times a week for the experimental groups of patients with chronic and acute mental disorders, and horticultural occupational therapy using a healing garden was provided once a week for the experimental groups. For the control groups of patients with acute and chronic mental disorders, occupational therapy was provided 5 times a week only.

\section{Research procedures}

For the experimental groups of patients with acute and chronic mental disorders, a horticultural occupational therapy program was provided once a week, a total of 12 sessions in a healing garden. For the emotional stability of patients with mental disorders, the horticultural occupational therapy program was conducted after performing activity analysis to ensure they understand activities and are suggested with proper levels of activities. The program was divided into 4 steps: motivation, performance, interaction and relaxation. To each step, 3 weeks were assigned, and, thus, the program was conducted for a total of 12 weeks. The first step (motivation) was composed of activities to ensure patients become interested in the program, and to change negative thoughts into positive ones. The second step (performance) was designed to encourage them to change their negative thoughts about themselves in the process of completing an assigned task. The third step (interaction) was composed of activities that can promote interactions with others in order to induce behavioral changes. The last step (relaxation) was designed to ease tension and stress. For the control groups, a group occupational program focusing on music, arts and social skills was provided (Table 1).

Table 1. Comparison program of horticultural occupation therapy (HOT) and occupational therapy (OT) performed to patients with mental disorder in this study.

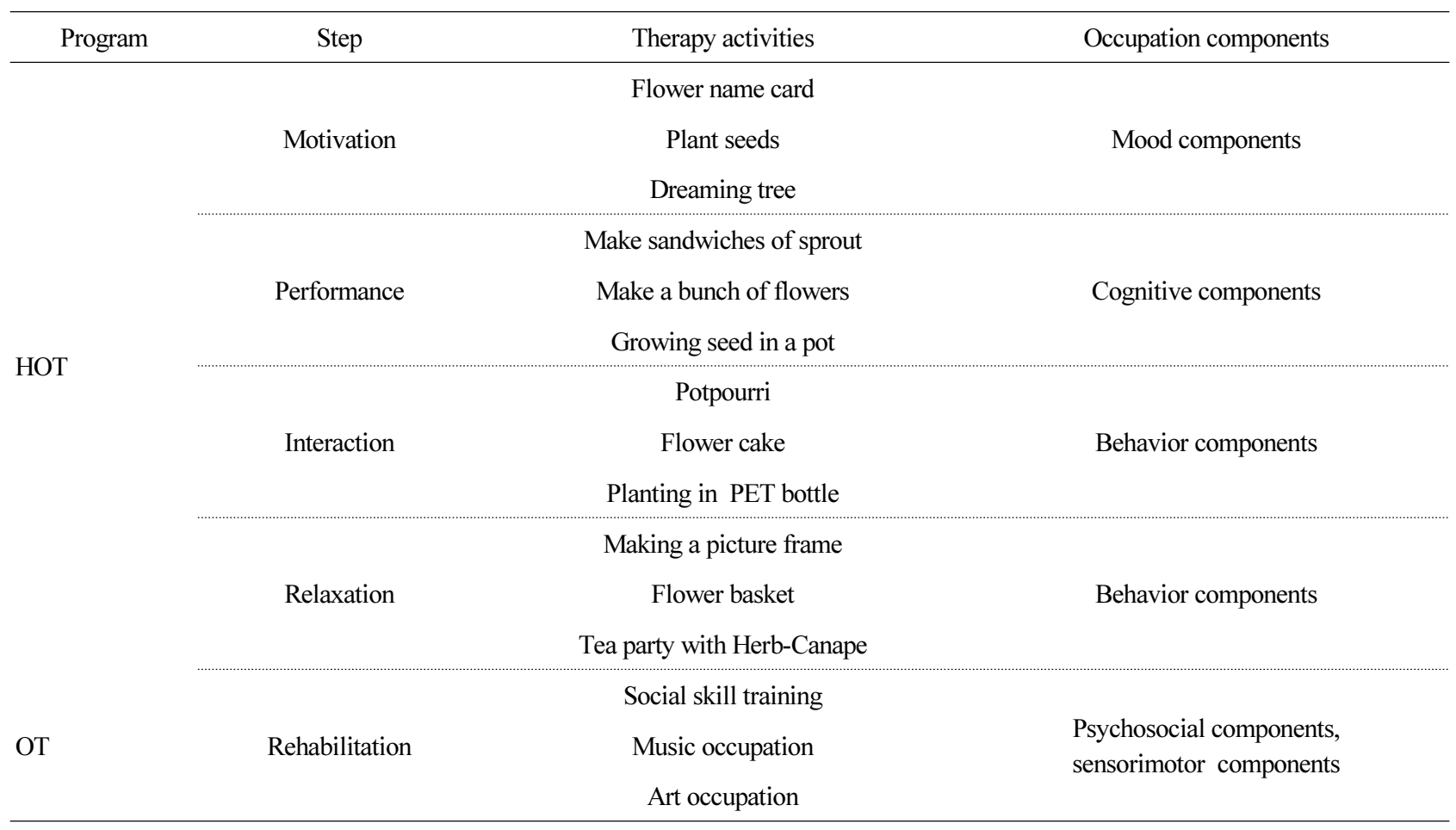




\section{Measurement tools}

\section{Emotional stress (ES)}

An emotional stress (ES) test is a self-report test performed to measure the level of stress, and 30 items of psychological abnormalities related to stress were selected from the Cornell Medical Index (CMI) and were reorganized considering the current conditions in Korea. Patients were instructed to give 2 points to each item if they have the symptom, and to give one point if they do not have the symptom. The lower the total score, the less physical symptoms they have (Brodman et al., 1949).

\section{Physical stress (PSE)}

A physical stress (PSE) test was performed using 30 items of physical abnormalities related to stress that were selected from the Cornell Medical Index (CMI) and reorganized considering the current conditions in Korea. Patients were instructed to give 2 points to each item if they have the symptom, and to give one point if they do not have the symptom. The lower the total score, the less physical symptoms they have (Brodman et al., 1949).

\section{Beck Depression Inventory (BDI)}

The Beck Depression Inventory (BDI) is a self-report scale that is used to evaluate the level of depression, and it is composed of 21 items on cognitive, emotional, motivational and physical symptoms related to depression. Each question is rated on a scale of 0 to 3, and, thus, the total score ranges from 0 to 63 points. The higher the total score, the higher the level of depression (Beck, 1967).

\section{Beck Anxiety Inventory (BAI)}

The Beck Anxiety Inventory (BAI) is a self-report scale that is used in the department of psychiatry to measure the level of anxiety, and it is composed of 21 items on cognitive, emotional and physical areas related to anxiety. Each question is rated on a scale of 0 to 3 , and, thus, the total score ranges from 0 to 63 points. This scale was developed to distinguish anxiety from depression (Beck et al., 1988).

\section{Analysis methods}

Demographic characteristics were examined using frequency analysis and SPSS PC 13.0. A paired $t$-test on the depression, stress and anxiety levels of the experimental and control groups before and after performing the horticultural occupational therapy program was conducted in order to verify the effects of the program. To identify differences in the effects of occupational therapy between the four groups of patients with chronic and acute mental disorders, a one-way ANOVA was conducted. The significance level was set as $p<.01$.

\section{Results and Discussion}

\section{General characteristics of research subjects}

The number of female patients was higher than that of male patients in all the groups of patients with acute and chronic mental disorders. In terms of age, the share of those in their 30s was the highest in the control group of patients with acute mental disorders (45\%) and the control group of patients with chronic mental disorders (40\%). The share of those in their 30 s was the highest in the control group of patients with chronic mental disorders (40\%), and the share of those in their 50s 
was the highest in the experimental group of patients with chronic mental disorders (45\%). Those who were diagnosed less than one year ago accounted for $100 \%$ in the group of patients with acute mental disorders. Those who were diagnosed earlier than 36 months ago accounted for $80 \%$ in the control group of patients with chronic mental disorders, and those who were diagnosed earlier than 13 months ago but less than 36 months ago accounted for $60 \%$ in the experimental group (Table 2).

\section{Effects of horticultural occupational therapy on acute mental disorders}

The average anxiety level of the experimental group of patients with acute mental disorders before the program was 34.09 points, which was reduced to 17.27 points after the program. Their average depression level before the program was 32.27 points, which was reduced to 18.91 points after the program. The results indicate that the program was effective in reducing the level of anxiety and depression, and there was a statistically significantly decrease. The average emotional stress (ES) score before the program was 29.18 points, which was reduced to 14.27 points after the program, showing a statistically significant decrease. The average physical stress (PSE) score before the program was 19.45 points, which was

Table 2. General characteristics of the patients participated in this study.

\begin{tabular}{|c|c|c|c|c|c|}
\hline & & \multicolumn{4}{|c|}{ No. of patients (\%) } \\
\hline \multicolumn{2}{|c|}{ General characteristics } & \multicolumn{2}{|c|}{ Acute } & \multicolumn{2}{|c|}{ Chronic } \\
\hline & & Control & Treatment & Control & Treatment \\
\hline \multirow{2}{*}{ Gender } & Male & $0(0)$ & $3(36)$ & $5(50)$ & $1(10)$ \\
\hline & Female & $11(100)$ & $8(64)$ & $5(50)$ & $9(90)$ \\
\hline \multirow{4}{*}{ Age } & $20-30$ & $2(18)$ & $1(9)$ & $2(20)$ & $1(10)$ \\
\hline & $31-40$ & $5(45)$ & $2(18)$ & $4(40)$ & $4(40)$ \\
\hline & $51-60$ & $2(18)$ & $5(45)$ & $2(20)$ & $5(50)$ \\
\hline & $61-80$ & $2(18)$ & $3(27)$ & $2(20)$ & $0(0)$ \\
\hline \multirow{3}{*}{$\begin{array}{l}\text { Duration } \\
\text { (month) }\end{array}$} & 12 & $11(100)$ & $11(100)$ & $0(0)$ & $0(0)$ \\
\hline & $13-36$ & $0(0)$ & $0(0)$ & $2(20)$ & $6(60)$ \\
\hline & $36-216$ & $0(0)$ & $0(0)$ & $8(80)$ & $4(40)$ \\
\hline
\end{tabular}

Table 3. Changes in acute mental disorder, Emotional stress scale (ES), Physical stress scale (PSE), Beck Anxiety Inventory (BAI) and Beck Depression Inventory (BDI) between pre and post occupational therapy (OT) and horticultural occupation therapy (HOT).

\begin{tabular}{|c|c|c|c|c|}
\hline Group & Measuring tool & Before Program & After Program & $\mathrm{P}$ \\
\hline \multirow{4}{*}{ Control } & ES & $25.64 \pm 10.93$ & $26.18 \pm 6.94$ & .135 \\
\hline & PSE & $22.73 \pm 9.55$ & $18.73 \pm 4.58$ & .278 \\
\hline & BAI & $29.0 \pm 12.68$ & $25.18 \pm 9.60$ & .400 \\
\hline & BDI & $30.18 \pm 11.20$ & $28.73 \pm 12.59$ & .260 \\
\hline \multirow{4}{*}{ Treatment } & ES & $29.18 \pm 10.52$ & $14.27 \pm 7.33$ & $.000 *$ \\
\hline & PSE & $19.45 \pm 7.32$ & $13.64 \pm 11.15$ & .108 \\
\hline & BAI & $34.09 \pm 9.10$ & $17.27 \pm 13.12$ & $.003 *$ \\
\hline & BDI & $32.27 \pm 10.95$ & $18.91 \pm 11.39$ & $.001^{*}$ \\
\hline
\end{tabular}

$* p<.01$ 
reduced to 13.64 points after the program. However, there was no statistically significant difference. Meanwhile, the control group of patients with acute mental disorders showed changes in their anxiety, depression, ES and PSE scores, but there was no statistically significant difference (Table 3). Patients with acute mental disorders tend to recover faster than those with chronic mental disorders, and to show many changes due to the effects of drugs they take. However, the fact that the group of patients with acute mental disorders who were provided with a general group occupational therapy program did not show any significant change in terms of their anxiety and depression indicates that horticultural occupational therapy was effective for the emotional recovery of patients with acute mental disorders along with drug treatment.

\section{Effects of horticultural occupational therapy on chronic mental disorders}

The average anxiety level of the experimental group of patients with chronic mental disorders before the program was 20.18 points, which was reduced to 16.73 points after the program. Their average depression level before the program was 22.73 points, which was reduced to 16.91 points after the program. The average emotional stress (ES) score before the program was 13.55 points, which was reduced to 12.18 points after the program, but there was no statistically significant difference. The average physical stress (PSE) score before the program was 13.73 points, which was reduced to 9.18 points after the program, showing a statistically significant difference. Meanwhile, the control group of patients with chronic mental disorders showed slight changes in their anxiety, depression, ES and PSE scores, but there was no statistically significance (Table 4). Patients with chronic mental disorders tend to be repeatedly hospitalized and discharged due to the characteristics of chronic mental disorders including the recurrences of the disorders, and it is difficult to expect drastic changes in their mental symptoms. That is why the illnesses are called chronic mental disorders. However, there was a significant decrease in the physical stress of patients with chronic mental disorders, which indicates that physical activities conducted in a healing garden naturally reduced their physical stress level.

\section{Comparison of the effects of horticultural occupational therapy on acute and chronic mental disorders}

The effects of horticultural occupational therapy on patents with acute and chronic mental disorders were compared, and the results show that the emotional stress (ES), anxiety and depression of patients with acute mental disorders were

Table 4. Changes in chronic mental disorder, Emotional stress scale (ES), Physical stress scale (PSE), Beck Anxiety Inventory (BAI) and Beck Depression Inventory (BDI) between pre and post occupational therapy (OT) and horticultural occupation therapy (HOT).

\begin{tabular}{ccccc}
\hline \multicolumn{1}{c}{ Group } & Measuring tool & Program Before & Program After & P \\
\hline \multirow{4}{*}{ Control } & ES & $6.82 \pm 7.04$ & $14.82 \pm 13.37$ & .116 \\
& PSE & $9.73 \pm 6.26$ & $9.64 \pm 7.08$ & .966 \\
& BAI & $28.09 \pm 16.8$ & $26.82 \pm 15.53$ & .053 \\
& BDI & $26.64 \pm 12.15$ & $27.64 \pm 12.08$ & .803 \\
\multirow{4}{*}{ Treatment } & ES & $13.55 \pm 10.53$ & $12.18 \pm 10.17$ & .285 \\
& PSE & $13.73 \pm 8.11$ & $9.18 \pm 7.96$ & $.001^{*}$ \\
& BAI & $20.18 \pm 19.48$ & $16.73 \pm 14.51$ & .150 \\
& BDI & $22.73 \pm 14.40$ & $16.91 \pm 11.36$ & .056 \\
\hline
\end{tabular}

$* p<.01$ 


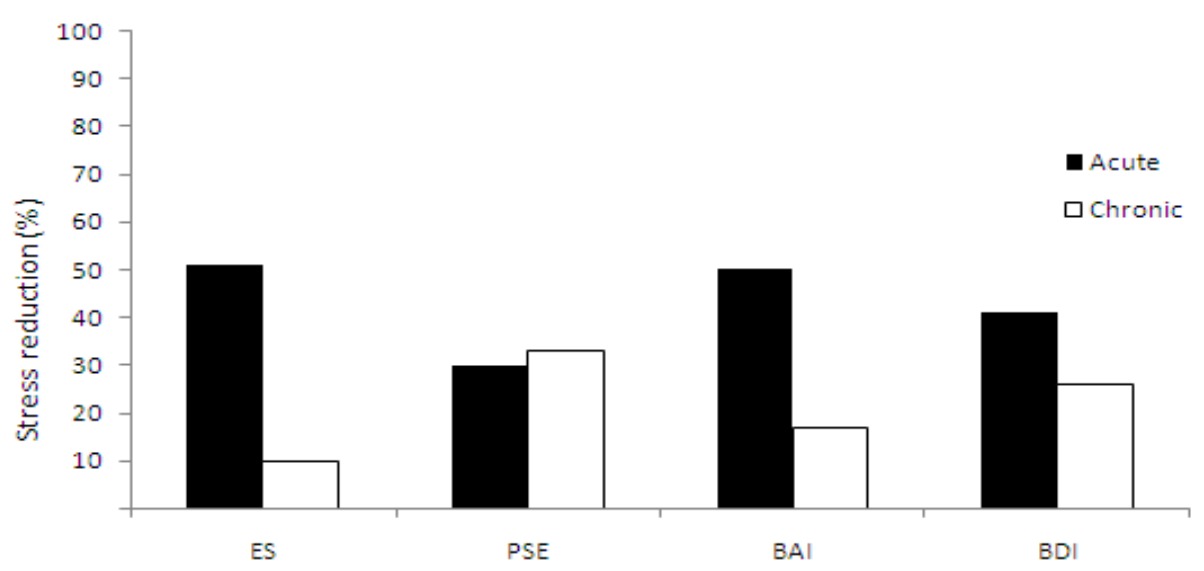

Figure 1. Comparison of post-horticultural occupational therapy for acute mental disorder and chronic mental disorder. $\mathrm{ES}=$ Emotional stress scale; PSE=Physical stress scale; BDI=Beck Depression Inventory; BAl=Beck Anxiety Inventory.

significantly reduced by $51 \%, 49.3 \%$ and $41.3 \%$ respectively, while those of patients with chronic mental disorders were reduced by $10.1 \%, 17.1 \%$ and $25.5 \%$ respectively. The results indicate that the program is more effective in improving the emotional status of patients with acute mental disorders (Fig. 1). However, the physical stress (PSE) of patients with chronic mental patients was reduced by $33.1 \%$, while that of patients with acute mental disorders was reduced only by $19.9 \%$, which indicates that the program was more effective in improving the physical status of patients with chronic mental disorders (Fig. 1). One of the main causes of mental illnesses is difficulties in adapting to social environments due to a high level of stress, and it is important to spend time doing meaningful activities that give a sense of satisfaction through work in order to overcome the difficulties (Meyer, 1992). The group of patients with chronic mental disorders who used a healing garden experienced many physical activities. The treatment space for them was extended from a hospital room to an outside space, that is, in this study, a healing garden, which offers nature in miniature, promotes interactions between humans and nature, and provides a space for social adaptation training. Since patients with acute mental disorders are likely to commit a suicide and escape, indoor programs are suitable for them. For patients with chronic mental disorders who have taken drugs for a long time and show no risk of committing a suicide and escape, making the most of a healing garden will be more effective.

Mental disorders are worsened or repeated due to a lack of interactions with intrinsic factors such as surrounding environments and stress, which makes social participation more difficult (Liberman, 1987). The horticultural occupational therapy program that was conducted to improve the skills of patients with mental disorders in overcoming difficulties and to reduce their vulnerability to stress focused on learning from and activities in environments, instead of intrinsic factors and used a healing garden as a treatment tool for motivation. Patients with mental disorders were induced to experience positive emotions as much as possible through horticultural activities, and to self-actualize through repetitive successes in assigned tasks. In the first step, activities such as making a name tag using pressed flowers, sowing, and making a tree of hope were performed to encourage participants to express their positive aspects, to think of hope through horticultural activities, and to motivate them as much as possible. Their participation rate was high, and they showed an active attitude in this step. The second step was designed to change their negative thoughts on themselves in the process of completing an assigned task, and, thus, to improve the performance of patients. Through the activities, patients who participated in the program were able to self-reinforce and have confidence in successfully performing something by maintaining or changing a certain behavior. By doing so, they were able to raise their voices and express themselves more. According to the cognitive behavioral theory, negative thoughts lower expectations of success and performance (Beck, 1976), while 
positive experiences, that is, positive emotional effects such as horticultural activities, make people have positive ideas about themselves and recognize their possibility, which results in performance and action. In the third step, manual activities such as making a herb pouch and a flower cake, and cutting trees were performed in order to promote interactions with others and thus to induce changes in behaviors. The fourth step was designed to ease tension and reduce stress. Interactions with others in the same group were promoted, and it was ensured that behaviors were controlled in order to reduce stress. The results of this study indicate that mental disorders need to be treated by reducing patients' symptoms through drug treatment, and, at the same time, by improving their ability to adapt to society through rehabilitation treatment, and thus to build their ability to respond to the risk of recurrence (Chu, 2007). It will be necessary to continue to research on multilateral treatment approaches to improve the effects of horticultural occupational therapy programs and to develop intermediate and advanced horticultural therapy programs.

\section{Conclusions}

This study aimed to analyze the effects of a horticultural vocational program using a healing garden on the emotional rehabilitation of patients with acute and chronic mental disorders, and to identify the possibility of using horticultural occupational therapy as a mental rehabilitation service to promote the social rehabilitation of patients with mental disorders. This study was conducted on 22 patients with acute mental disorders and 20 patients with chronic mental patients, and they were divided into the experimental and control groups as they preferred regardless of their gender and age. General occupational therapy was provided 4 times a week for the experimental groups of patients with chronic and acute mental disorders, and a horticultural occupational therapy program using a healing garden was provided only once a week, a total of 12 sessions, for the experimental groups. For the control groups of patients with acute and chronic mental disorders, occupational therapy was provided 5 times a week only. Their emotional stress (ES), physical stress (PSE), depression and anxiety were measured and changes before and after performing this experiment were analyzed. The horticultural occupational therapy treatment used to promote social rehabilitation and reduce mental symptoms was found to be more effective in reducing ES, depression and anxiety in the experimental group of patients with acute mental disorders who participated in horticultural activities, compared to the control group. In addition, the PSE of the group of patients with chronic mental disorders who participated in horticultural occupational therapy was reduced more, compared to that of the control group.

\section{References}

Beck, A.T. 1967. Depression: Clinical, Experimental, and Theoretical Aspects. New York, NY: Hoeber Medical Division, Harper \& Row.

Beck, A.T. 1976. Cognitive Therapy and Emotional Disorders. New York, NY: International Universities Press.

Beck, A.T., N. Epstein, G. Brown, and R.A. Steer. 1988. An Inventory for Measuring Clinical Anxiety: Psychometric Properties. J. Consult. Clin. Psychol. 56(6):893-897. DOI:10.1037/0022-006X.56.6.893

Brodman, K., A.J. Erdmann Jr., I. Lorge, H.G. Wolff, and T.H. Broadbent. 1949. The Cornell Medical Index : An adjunct to medical interview. J. Am. Med. Assoc. 140(6):530-534. DOI:10.1001/jama.1949.02900410026007

Chu, K.J. 2007. The effect of horticultural therapy on anxiety and self-esteem for patients with schizophrenia. MS thesis, Jinju National University, Jinju, Korea.

Doxon, L.E., R.H. Mattson, and A.P. Jurich. 1987. Human stress reduction through horticultural vocation training. HortScience 22(4):655-656. 
Goldman, H.H., A.A. Gattozzi, and C.A. Taube. 1981. Defining and counting the chronically mentally ill. Hosp. Community Psychiatry 32(1):21-27. DOI:10.1176/ps.32.1.21

Lee, M.S., K.S. Lee, K.O. Oh, and J.H. Ku. 2006. Effect of horticultural therapy on behavioral psychotic symptom and sociability of schizophrenic patients. J. Korean Soc. People Plants Environ. 9(4):130-139.

Liberman, R.P. 1987. Psychological intervention in the management of schizophrenia : Overcoming disability and handicap. Presented at the American Psychiatric Symp. On Current Problems and Strangers for the treatment of schizophrenia, Chicago. USA.

Marcus, C.C. and M. Barnes. 1999. Healing Gardens: Therapeutic Benefits and Design Recommendations. New York, NY: John Wiley and Sons.

Meyer, A. 1922. The philosophy of occupation therapy. Arch. Occup. Ther. 1:1-10.

Park, Y.S. 1995. The effects of systematic group music therapy for the inpatients with depressive mood and psychiatric behavior. MS Thesis, Seoul National University, Seoul, Korea.

Relf, P.D. 1990. Psychological sociological response to plants : Implications for horticulture. HortScience 25(1):11-13.

Um, S.J. 2000. Effect of horticultural therapy on the changes of self-esteem and sociality of the chronic schizophrenia. MS Thesis. Kunkuk university, Seoul, Korea. 